\title{
(D) crossMark \\ Higher interleukin-6 levels and changes in transforming growth factor- $\beta$ are associated with lung impairment in pulmonary tuberculosis
}

To the Editor:

Pulmonary tuberculosis (PTB) is associated with granuloma formation, necrosis and cavitation in lung tissue. Lung injury in PTB can persist despite microbiological cure and is associated with COPD independent of smoking exposure [1]. Furthermore, pulmonary sequelae of PTB are an under-recognised cause of respiratory disability and excess mortality [2].

Our previous work in India found up to $50 \%$ of PTB cases had impaired lung function post-treatment [3]. We also found a positive correlation between the duration of symptomatic illness prior to PTB treatment and severity of lung impairment post-treatment. These data suggest that acute lung injury in PTB probably occurs during the pre- or early-treatment period, and that lung tissue repair and remodelling during treatment may play an important role in post-PTB lung disease.

However, the pathogenesis of PTB-associated lung disease is unclear. Pre-clinical studies have suggested a pro-fibrotic role of interleukin (IL)-1 $\beta$, IL-6, IL-4, IL-17 and transforming growth factor (TGF)- $\beta$ s in the lungs $[4,5]$; markers that also play an important role in the host immune response in PTB. Further, tumour necrosis factor (TNF)- $\alpha$ and matrix metalloproteinases (MMPs) have been implicated in lung tissue destruction and cavitation in PTB [6].

There is growing interest in the utility of host directed therapies (HDTs); adjunctive therapies that modulate immune mechanisms in the host, for improving clinical outcomes. HDTs with anti-inflammatory properties could potentially prevent or limit the extent of lung injury in PTB. Therefore, we measured the association of potentially modifiable inflammatory markers implicated in lung tissue destruction and fibrosis, with respiratory morbidity and impaired lung function in a prospective cohort of adults with drug-sensitive PTB.

We randomly selected adults (18 years and older) with microbiologically confirmed PTB, receiving standard multidrug therapy in the CTRIUMPH study in India [7]. Participants with drug-resistant disease, previous PTB or previous chronic lung diseases were excluded. PTB cases were prospectively evaluated at initiation, 2 months and completion of treatment for respiratory health status using the Saint George's Respiratory Questionnaire (SGRQ). Pre- and post-bronchodilator spirometry was performed at treatment completion according to European Respiratory Society/American Thoracic Society guidelines [8]. Forced expiratory volume in $1 \mathrm{~s}\left(\mathrm{FEV}_{1}\right)$, forced vital capacity $(\mathrm{FVC})$ and the $\mathrm{FEV}_{1}$ to $\mathrm{FVC}$ ratio $\left(\mathrm{FEV}_{1} / \mathrm{FVC}\right)$ were z-score standardised for analysis using Global Lung Initiative reference equations [9].

Plasma samples collected at initiation, at 2 months and at completion of treatment were tested, in duplicate, for TNF- $\alpha$, IL-1 $\beta$, IL-4, IL-6, IL-17, TGF- $\beta 1$, TGF- $\beta 2$, TGF- $\beta 3$, MMP-1, MMP-3, MMP-7 and tissue inhibitor of MMPs (TIMP)-1, TIMP-2, TIMP-3 and TIMP-4 concentrations at the National

@ERSpublications

Higher levels of IL-6 and slow-to-resolve TGF- $\beta$ are associated with lung impairment in treated tuberculosis. These results have important implications for clinical trials of immunomodulatory therapies to prevent tuberculosis-associated lung disease. https://bit.ly/2FQEtsz

Cite this article as: Gupte AN, Selvaraju S, Gaikwad S, et al. Higher interleukin-6 levels and changes in transforming growth factor- $\beta$ are associated with lung impairment in pulmonary tuberculosis. ERJ Open Res 2021; 7: 00390-2020 [https://doi.org/10.1183/23120541.00390-2020].

Copyright $\odot$ ERS 2021. This article is open access and distributed under the terms of the Creative Commons Attribution NonCommercial Licence 4.0. 
Institutes of Health (NIH) - National Institute for Research in Tuberculosis - International Center for Excellence in Research, Chennai, India using multiplex ELISA (Bio-Rad Laboratories, CA, USA) on a Luminex platform using manufacturer recommended protocols. We used uni- and multivariable random effects regression to measure the pooled association between $\log _{2}$-transformed cytokine concentrations and total SGRQ scores during treatment. A difference of four points or more in the total SGRQ score was considered clinically relevant [10]. Uni- and multivariable linear regression and Spearman's correlation coefficient were used to measure the association of cytokine concentrations, and their change during treatment, with post-bronchodilator lung function at treatment completion. Multivariable regression analyses accounted for confounding by age, sex, smoking exposure, diabetes and markers of PTB disease severity, including body mass index (BMI), smear grade, cavitary disease and duration of symptomatic illness prior to treatment initiation. The p-values were adjusted for multiple comparisons using the Benjamini-Hochberg procedure and a $10 \%$ false discovery rate.

We enrolled 30 PTB cases contributing 90 person-visits from the CTRIUMPH study. Participants selected for this analysis were comparable with those not selected in terms of their baseline characteristics. Overall, $20(74 \%)$ were male, 9 (31\%) ever-smoked, 2 (7\%) had HIV coinfection, 7 (26\%) had diabetes and 11 (37\%) had cavitation on chest radiography. The median (interquartile range) age and BMI was 36 (28-50) years and $18.1(16.0-20.0) \mathrm{kg} \cdot \mathrm{m}^{-2}$, respectively. Cytokine concentrations did not differ significantly by cavitary disease, sputum smear grade or BMI.

Cytokine concentrations at treatment initiation were not associated with lower lung function at treatment completion. All cytokine concentrations declined during treatment. However, greater declines in TGF- $\beta 2$ during treatment were associated with higher $\mathrm{FEV}_{1} / \mathrm{FVC} \mathrm{z}$ scores post-treatment. Specifically, greater declines in TGF- $\beta 2$ during the first 2 months of treatment, but not the last 4 months, were associated with higher $\mathrm{FEV}_{1} / \mathrm{FVC}$ post-treatment (0.78-point higher $\mathrm{z}$ score per twofold decline in TGF- $\beta 2$, $95 \%$ CI 0.28 to $1.29, \mathrm{p}=0.005)$ in the univariable analysis. After adjusting for potential confounders, including markers of disease severity, a twofold decline in TGF- $\beta 2$ was associated with a 0.80 -point higher z score $(95 \% \mathrm{CI}$ 0.30 to $1.30, \mathrm{p}=0.005)$. Interestingly, $\mathrm{PTB}$ cases in the lowest tertile of $\mathrm{FEV}_{1} / \mathrm{FVC} \mathrm{z}$ scores at treatment completion had a paradoxical increase in TGF- $\beta 2$ levels during the first 2 months of treatment compared to PTB cases with $\mathrm{FEV}_{1} / \mathrm{FVC} \mathrm{z}$ scores in the highest tertile $(\mathrm{p}=0.005)$ (figure 1a).

The median (interquartile range) SGRQ score was 43 (29-55) points at treatment initiation and declined to $6(3-19)$ points at treatment completion $(\mathrm{p}<0.001)$ (figure $1 \mathrm{~b}$ ). Higher levels of IL-6 were associated with higher SGRQ scores during treatment (three-point-higher SGRQ score per twofold higher IL-6 concentrations; 95\% CI 2 to 5, p=0.002) in the univariable analysis. After adjusting for potential confounders, including markers of disease severity, a twofold higher IL-6 concentration was associated with a clinically relevant 4-point higher SGRQ score (95\% CI 1 to 5, p=0.004) during treatment.
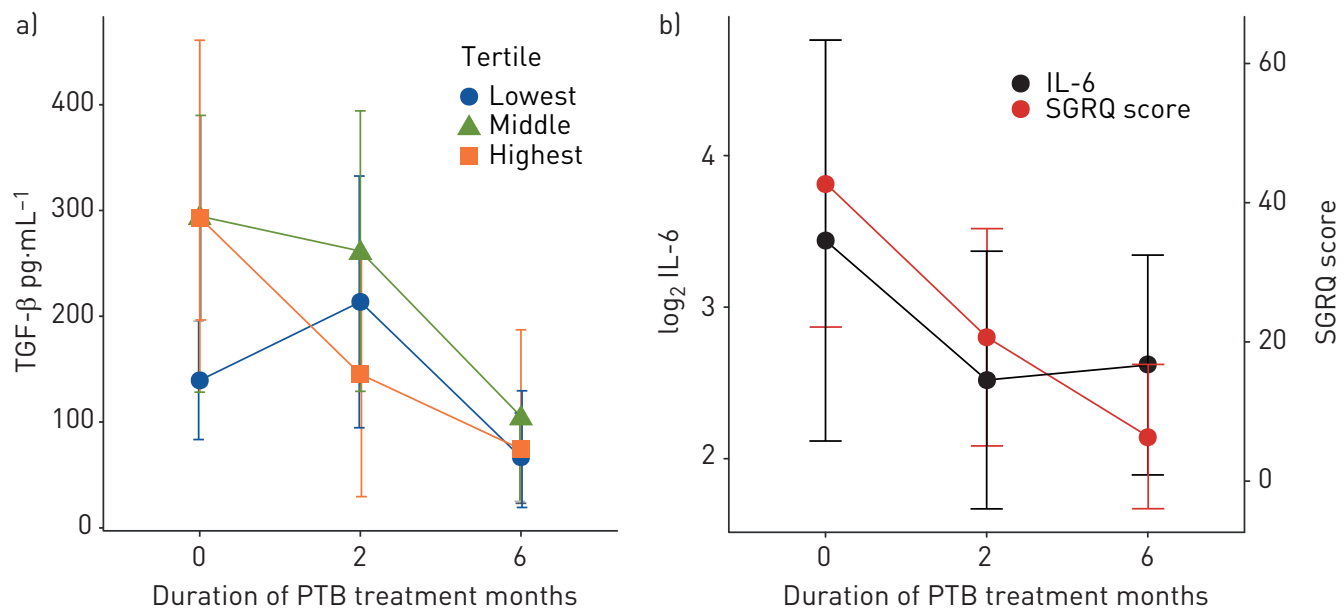

FIGURE 1 a) Trends in transforming growth factor (TGF)- $\beta 2$ during pulmonary tuberculosis (PTB) treatment stratified by tertiles of forced expiratory volume in $1 \mathrm{~s}\left(\mathrm{FEV}_{1}\right) /$ forced vital capacity $(\mathrm{FVC}) \mathrm{z}$-scores at treatment completion. $\mathrm{FEV}_{1} / \mathrm{FVC} \mathrm{z}$-score thresholds for the lowest and middle tertiles were -0.62 and 0.10 , respectively. Relative to PTB cases with $\mathrm{FEV}_{1} / \mathrm{FVC}$ z-scores in the highest tertile at treatment completion, those in the lowest tertile had a paradoxical increase in TGF- $\beta 2$ concentrations during the first 2 months of treatment. b) Trends in interleukin (IL)-6 and total St George's Respiratory Questionnaire (SGRQ) scores during PTB treatment. The median SGRQ score declined from 43 points at treatment initiation to 6 points at treatment completion, and correlated with IL-6 concentrations during PTB treatment. 
We did not find a statistically significant association between TNF- $\alpha$, IL-1 $\beta$, IL-4, IL-17, MMP-1, MMP-3, MMP-7, TIMP-1, TIMP-2, TIMP-3 and TIMP-4 and total SGRQ scores or impaired lung function in our cohort.

TGF- $\beta$ has been implicated in pulmonary fibrosis and airway remodelling through cellular growth and differentiation [11]. Pre-clinical studies have described the role of TGF- $\beta$ in granuloma formation in PTB [12]. A novel finding of our study was the association between greater declines in TGF- $\beta 2$ levels during early PTB treatment and better lung function post-treatment. Importantly, PTB patients in the lowest tertile of lung function post-treatment had a paradoxical increase in TGF- $\beta 2$ levels during the first 2 months of treatment. These data support the role of TGF- $\beta$ in post-PTB lung impairment and suggest that facilitating rapid resolution of TGF- $\beta$ through immunomodulatory HDTs during early treatment may mitigate post-PTB lung sequelae.

Previous studies in patients with COPD have demonstrated an inverse relationship between IL- 6 and functional capacity [13]. However, the role of IL-6 in PTB-associated respiratory morbidity has not been studied. We have previously shown that poor respiratory health status was associated with all-cause mortality in PTB [14]. Here, we report that patients with PTB with higher IL-6 levels had worse respiratory health status during treatment. While these data suggest a role of IL-6 modulation for reducing respiratory morbidity in PTB, the impact of elevated IL-6 levels on long-term lung impairment and mortality needs further investigation.

Our single-cohort study was limited by its small sample size and was probably underpowered to detect clinically relevant associations of a smaller magnitude. Well-powered validation studies in independent cohorts are needed to confirm our study findings. We additionally did not measure lung function at PTB treatment initiation or assess progression of post-PTB lung impairment. Despite these limitations, we report a novel association of elevated IL- 6 and slow-to-resolve TGF- $\beta$, with lung impairment in PTB. Given the availability of US Food and Drug Administration and European Medicines Agency-approved IL-6 and TGF- $\beta$ inhibitors, our study findings have potentially important implications for the optimal timing and immune targets of future HDT trials to prevent PTB-associated lung disease.

Akshay N. Gupte ${ }^{1,2}$, Sriram Selvaraju ${ }^{3}$, Sanjay Gaikwad ${ }^{4}$, Vidya Mave ${ }^{1,2}$, Pavan Kumar ${ }^{5}$, Subash Babu ${ }^{5}$, Bruno B. Andrade $\odot^{6,7,8}$, William Checkley ${ }^{9}$, Robert Bollinger ${ }^{1,2}$ and Amita Gupta ${ }^{1,2}$ for the CTRIUMPH study team

${ }^{1}$ Division of Infectious Diseases, Johns Hopkins University School of Medicine, Baltimore, MD, USA.

${ }^{2}$ Center for Clinical Global Health Education, Johns Hopkins University School of Medicine, Baltimore, MD, USA. ${ }^{3}$ Dept of Clinical Research, National Institute for Research in Tuberculosis, Chennai, India. ${ }^{4}$ Dept of Pulmonary Medicine, Byramjee-Jeejeebhoy Government Medical College, Pune, India. ${ }^{5}$ National Institutes of Health - National Institute for Research in Tuberculosis - International Center for Excellence in Research, Chennai, India. ${ }^{6}$ Instituto Gonçalo Moniz, Fundação Oswaldo Cruz (FIOCRUZ), Salvador, Brazil. ${ }^{7}$ Multinational Organisation Network Sponsoring Translational and Epidemiological Research (MONSTER) Initiative, Salvador, Brazil. ${ }^{8}$ Universidade Salvador (UNIFACS), Laureate Universities, Salvador, Brazil. ${ }^{9}$ Division of Pulmonary Medicine, Johns Hopkins University School of Medicine, Baltimore, MD, USA.

Correspondence: Akshay N. Gupte, Division of Infectious Diseases, Johns Hopkins University School of Medicine, 600 N. Wolfe Street, Baltimore, Maryland 21205-2196, USA. E-mail: agupte1@jhmi.edu

Received: 15 June 2020 | Accepted after revision: 16 Sept 2020

Acknowledgements: We thank Artur Queiroz (FIOCRUZ and MONSTER Initiative, Salvador, Brazil) for designing the figures in this manuscript.

Conflict of interest: A.N. Gupte has nothing to disclose. S. Selvaraju has nothing to disclose. S. Gaikwad has nothing to disclose. V. Mave has nothing to disclose. P. Kumar has nothing to disclose. S. Babu has nothing to disclose B.B. Andrade has nothing to disclose. W. Checkley has nothing to disclose. R. Bollinger reports grants from US NIH during the conduct of the study; and nonfinancial support from Hologic Inc., Merck \& Company, and Emocha Health, outside the submitted work. A. Gupta reports grants from NIH during the conduct of the study.

Support statement: Data in this manuscript were collected as part of the Regional Prospective Observational Research for Tuberculosis (RePORT) India Consortium. This project has been funded with Federal funds from the Government of India's Dept of Biotechnology (DBT), the Indian Council of Medical Research (ICMR), the US NIH, National Institute of Allergy and Infectious Diseases (NIAID), and Office of AIDS Research, and distributed in part by CRDF Global. Research reported in this publication was also supported by NIAID of the NIH under award numbers R01AI097494 and UM1AI069465, the Wyncote Foundation, the Gilead Foundation and the Ujala Foundation, Newton Square, PA, USA. This research was also funded, in part, by a 2019 developmental grant from the Johns Hopkins 
University Center for AIDS Research, an NIH-funded programme (1P30AI094189), which is supported by the following NIH Co-Funding and Participating Institutes and Centers: NIAID, NCI, NICHD, NHLBI, NIDA, NIA, NIGMS, NIDDK and NIMHD. The content is solely the responsibility of the authors and does not necessarily represent the official views of the NIH. A.N. Gupte was supported by NIH Research Training Grant number D43 TW009340 funded by the NIH Fogarty International Center, NINDS, NIMH, NHBLI and NIEHS. The funders had no role in study design; in the collection, analysis and interpretation of data; in the writing of the report; or in the decision to submit the article for publication. The contents of this publication are solely the responsibility of the authors, and do not represent the official views of the DBT, the ICMR, the NIH or CRDF Global. Any mention of trade names, commercial projects or organisations does not imply endorsement by any of the sponsoring organisations. The authors also acknowledge support from Persistent Systems in kind. Funding information for this article has been deposited with the Crossref Funder Registry.

\section{References}

1 Byrne AL, Marais BJ, Mitnick CD, et al. Tuberculosis and chronic respiratory disease: a systematic review. Int $J$ Infect Dis 2015; 32: 138-146.

2 Pasipanodya JG, McNabb SJ, Hilsenrath P, et al. Pulmonary impairment after tuberculosis and its contribution to TB burden. BMC Public Health 2010; 10: 259.

3 Gupte AN, Paradkar M, Selvaraju S, et al. Assessment of lung function in successfully treated tuberculosis reveals high burden of ventilatory defects and COPD. PLoS ONE 2019; 14: e0217289.

4 Fielding CA, Jones GW, McLoughlin RM, et al. Interleukin-6 signaling drives fibrosis in unresolved inflammation. Immunity 2014; 40: 40-50.

5 Duan FF, Barron G, Meliton A, et al. P311 promotes lung fibrosis via stimulation of transforming growth factor-beta1, -beta2, and -beta3 translation. Am J Respir Cell Mol Biol 2019; 60: 221-231.

6 Ong CW, Elkington PT, Friedland JS. Tuberculosis, pulmonary cavitation, and matrix metalloproteinases. Am J Respir Crit Care Med 2014; 190: 9-18.

7 Gupte A, Padmapriyadarsini C, Mave V, et al. Cohort for Tuberculosis Research by the Indo-US Medical Partnership (CTRIUMPH): protocol for a multicentric prospective observational study. BMJ open 2016; 6: $\mathrm{e} 010542$.

8 Miller MR, Hankinson J, Brusasco V, et al. Standardisation of spirometry. Eur Respir J 2005; 26: 319-338.

9 Quanjer PH, Hall GL, Stanojevic S, et al. Age- and height-based prediction bias in spirometry reference equations. Eur Respir J 2012; 40: 190-197.

10 Jones PW, Beeh KM, Chapman KR, et al. Minimal clinically important differences in pharmacological trials. Am J Respir Crit Care Med 2014; 189: 250-255.

11 Tatler AL, Jenkins G. TGF-beta activation and lung fibrosis. Proc Am Thorac Soc 2012; 9: 130-136.

12 DiFazio RM, Mattila JT, Klein EC, et al. Active transforming growth factor-beta is associated with phenotypic changes in granulomas after drug treatment in pulmonary tuberculosis. Fibrogenesis Tissue Repair 2016; 9: 6.

13 Walter RE, Wilk JB, Larson MG, et al. Systemic inflammation and COPD: the Framingham Heart Study. Chest 2008; 133: 19-25.

14 Gupte AN, Selvaraju S, Paradkar M, et al. Respiratory health status is associated with treatment outcomes in pulmonary tuberculosis. Int J Tuberc Lung Dis 2019; 23: 450-457. 